\title{
Hsa-miR-371-5p inhibits human mesangial cell proliferation and promotes apoptosis in lupus nephritis by directly targeting hypoxia-inducible factor $1 \alpha$
}

\author{
FEIFEI YAO, LIQIU SUN, WEI FANG, HUAMIN WANG, DONGSHENG YAO, \\ RUI CUI, JIA XU, LI WANG and XIUMEI WANG
}

Department of Nephrology, The Fourth Hospital of Harbin Medical University, Harbin, Heilongjiang 150001, P.R. China

Received September 6, 2015; Accepted September 16, 2016

DOI: $10.3892 / \mathrm{mmr} .2016 .5939$

\begin{abstract}
MicroRNAs (miRNAs/miR) have emerged as a novel class of gene expression modulators in kidney disease. Lupus nephritis (LN) is the predominant cause of morbidity and mortality in patients with systemic lupus erythematosus (SLE). Hsa-miR-371-5p has previously been reported to be dysregulated in LN using a miRNA microarray analysis. The present study aimed to determine the function and molecular mechanisms of hsa-miR-371-5p in human mesangial cells of LN. Quantitative polymerase chain reaction (qPCR) was used to detect hsa-miR-371-5p expression in LN tissues. Furthermore, the MTT assay and flow cytometric analyses were performed to analyze the effects of hsa-miR-371-5p on mesangial cell proliferation and apoptosis. Bioinformatics analysis, luciferase reporter assay, qPCR and western blotting were also conducted to predict and confirm the target gene of hsa-miR-371-5p in mesangial cells. The results demonstrated that hsa-miR-371-5p expression was markedly downregulated in LN renal tissues compared with in normal kidney tissues. Restoration of hsa-miR-371-5p expression using synthetic hsa-miR-371-5p mimics was able to significantly inhibit mesangial cell proliferation and induce apoptosis. In addition, mechanistic exploration demonstrated that hypoxia-inducible factor $1 \alpha(\mathrm{HIF}-1 \alpha)$ was a direct target gene of hsa-miR-371-5p in mesangial cells. In conclusion, these results suggested that hsa-miR-371-5p is downregulated in LN, and overexpression of hsa-miR-371-5p may inhibit mesangial cell proliferation and promote apoptosis by directly targeting HIF- $1 \alpha$.
\end{abstract}

Correspondence to: Professor Xiumei Wang, Department of Nephrology, The Fourth Hospital of Harbin Medical University, 37 Yiyuan Street, Harbin, Heilongjiang 150001, P.R. China E-mail: wxmhaerbing2010@163.com

Key words: lupus nephritis, hsa-miR-371-5p, proliferation and apoptosis, hypoxia inducible factor- $1 \alpha$

\section{Introduction}

Systemic lupus erythematosus (SLE) is a complex autoimmune disease, which is characterized by a loss of self-tolerance and the production of autoantibodies against a host of nuclear self-antigens (1). SLE predominantly affects women of childbearing age, and $\leq 20 \%$ of cases begin during teenage years. Lupus nephritis (LN) is kidney inflammation caused by SLE, and is the leading cause of morbidity and mortality in patients with SLE (2). One of the primary characteristics of LN is excessive proliferation of mesangial cells; disordered mesangial cell proliferation usually results in nephritic syndrome, which eventually leads to renal failure $(3,4)$. LN is a severe disorder and the currently available therapeutic methods are ineffective; therefore, it is essential to identify novel useful targets for the treatment of LN.

MicroRNAs (miRNAs/miR) are small, non-coding RNAs that endogenously control gene expression by partial complementary binding to the 3 ' untranslated region (3'-UTR) of target mRNAs (5-8). Previous studies have reported that miRNAs are involved in diverse physiological and pathophysiological functions, including cell differentiation and development, cell cycle and apoptosis, immune tolerance and carcinogenesis $(9,10)$. In addition, studies have demonstrated that several miRNAs exhibit aberrant expression in $\mathrm{LN}$. Dai et al identified 36 upregulated and 30 downregulated miRNAs in LN renal tissues compared with in healthy kidney tissues (11). Denby et al reported that miR-21 and miR-214 were induced by transforming growth factor- $\beta$ stimulation in an anti-Thy1.1 rat model (12). Furthermore, Te et al revealed that five miRNAs (hsa-miR-371-5p, hsa-miR-423-5p, hsa-miR-638, hsa-miR-1224-3p and hsa-miR-663) were abnormally expressed in LN across various racial groups, as determined by miRNA microarray analysis (13). These results suggest that miRNA may function as a valuable novel tool for understanding, diagnosing and treating $\mathrm{LN}$.

Hsa-miR-371-5p is a novel miRNA that is present on chromosome 19q13.42 in the human genome, the function of which has not yet been elucidated. Hsa-miR-371-5p has previously been reported to be dysregulated in LN (13). However, at present, the biological role and molecular mechanisms of hsa-miR-371-5p in LN remain unclear. 
The present study aimed to identify the biological function and molecular mechanisms of hsa-miR-371-5p in LN. Hsa-miR-371-5p was shown to be downregulated in LN tissues. Furthermore, exogenous restoration of hsa-miR-371-5p expression was able to inhibit human mesangial cell proliferation and promote apoptosis by directly targeting hypoxia-inducible factor $1 \alpha$ (HIF-1 $\alpha)$. The results of the present study indicate that hsa-miR-495 may be considered a potential therapeutic target for the future treatment of LN.

\section{Materials and methods}

Cell culture. The human mesangial cell line was obtained from the Cell Resource Center, Shanghai Institutes for Biological Sciences (Shanghai, China). The cells were cultured in Dulbecco's modified Eagle's medium (Invitrogen; Thermo Fisher Scientific, Inc., Waltham, MA, USA) supplemented with $10 \%$ fetal bovine serum (Beyotime Institute of Biotechnology, Haimen, China) at $37^{\circ} \mathrm{C}$ in an atmosphere containing $5 \% \mathrm{CO}_{2}$. Penicillin $(100 \mathrm{U} / \mathrm{ml})$ and streptomycin $(100 \mu \mathrm{g} / \mathrm{ml})$ were added to the culture medium to prevent bacterial contamination.

Renal samples. A total of 35 SLE renal biopsy samples were collected from the Fourth Hospital of Harbin Medical University (Harbin, China) between January 2010 and January 2014. All SLE patients (6 male and 29 female; mean age, 26.6 43.4 ) exhibited the necessary criteria for a diagnosis of SLE. The classification of LN was based on the International Society of Nephrology/Renal Pathology Society criteria published in 2003 (14). Renal carcinoma-adjacent normal kidney tissues were collected from 35 patients as the control group (18 male and 17 female; mean age, 47.6 \pm 4.7 ). Written informed consent was obtained from all of the participants or their relatives. The present study was approved by the Ethics Committee of the Fourth Hospital of Harbin Medical University, and was conducted in compliance with the Helsinki Declaration.

Total RNA extraction and reverse transcription-quantitative polymerase chain reaction $(R T-q P C R)$. Total RNA was isolated from homogenized SLE renal biopsy tissues, normal kidney tissues and mesangial cells using TRIzol ${ }^{\circledR}$ reagent (Invitrogen; Thermo Fisher Scientific, Inc.) according to the manufacturer's protocol. Subsequently, RNA was reverse transcribed using a miScript RT kit (Qiagen, Inc., Valencia, CA, USA) or a PrimeScript RT reagent kit (Takara Bio, Inc., Otsu, Japan) according to the manufacturer's protocols. qPCR was conducted using a TaqMan ${ }^{\circledR}$ miRNA assay kit (Applied Biosystems; Thermo Fisher Scientific, Inc.). qPCR was performed on an Applied Biosystems Prism ${ }^{\circledR} 7900 \mathrm{HT}$ sequence detection system (Applied Biosystems; Thermo Fisher Scientific, Inc.) with a total volume of $10 \mu$ I PCR mix, which consisted of: $1.0 \mu \mathrm{l}$ template cDNA, $4 \mu \mathrm{l}$ SYBR Green mixture and $1.0 \mu 1$ primers; the PCR mix was supplemented with water to adjust the final volume to $20 \mu \mathrm{l}$.

The hsa-miR-371-5p,U6, HIF-1 $\alpha$ and GAPDH primers were purchased from Applied Biosystems (Thermo Fisher Scientific, Inc.). The sequences were as follows: Hsa-miR-371-5p, forward 5'-ACTCAAAAGATGGCGGCAC-3', the reverse primer was supplied by the miScript RT kit; U6, forward 5'-CTC GCTTCGGCAGCACA-3', reverse 5'-ACGCTTCACGAA
TTTGCGT-3'; HIF-1 $\alpha$, forward 5'-CTCAGAATGAAGTGT ACCCTAA-3', reverse 5'-CAAATCAGCACCAAGCAG-3'; and GAPDH, forward 5'-GACCTGACCTGCCGTCTA-3', reverse 5'-AGGAGTGGGTGTCGCTGT-3'. All reactions were incubated in a 96 -well plate at $95^{\circ} \mathrm{C}$ for $20 \mathrm{~min}$, followed by 45 cycles at $95^{\circ} \mathrm{C}$ for $10 \mathrm{sec}$ and $60^{\circ} \mathrm{C}$ for $20 \mathrm{sec}$. The U6 gene was used as an internal control to normalize differences in hsa-miR-371-5p expression in each sample. The relative amount of hsa-miR-371-5p expression to U6 expression was determined using the $2^{-\Delta \Delta \mathrm{Cq}}(15)$.

Western blot analysis. Total protein was extracted from the cultured cells by using radioimmunoprecipitation assay buffer (Invitrogen; Thermo Fisher Scientific, Inc.). The concentration of each protein was detected by a Enhanced bicinchoninic acid Protein Assay kit (Beyotime Institute of Biotechnology). Equal amounts of protein $(40 \mu \mathrm{g})$ isolated from mesangial cells were separated by $10 \%$ SDS-PAGE and were blotted onto a polyvinylidene difluoride membrane (Merck Millipore, Darmstadt, Germany). After blocking with 5\% non-fat milk for $2 \mathrm{~h}$ at $37^{\circ} \mathrm{C}$, the membrane was incubated with mouse monoclonal anti-HIF-1 $\alpha$ (cat. no. ab113642; 1:1,000; Abcam, Cambridge, UK) and GAPDH (cat. no. ab8245; 1:10,000; Abcam) antibodies overnight at $4^{\circ} \mathrm{C}$. Subsequently, the membrane was washed three times with TBS containing $0.1 \%$ Tween for $15 \mathrm{~min}$ and was incubated with the corresponding horseradish peroxidase-conjugated secondary antibody (cat. no. ab6789; 1:2,000; Abcam) for $1 \mathrm{~h}$ at room temperature. The results were visualized by enhanced chemiluminescence (Merck Millipore).

Cell transfection assay. The synthesized hsa-miR-371-5p mimics and control mimics (Shanghai GenePharma Co., Ltd., Shanghai, China) were transfected into mesangial cells using Lipofectamine ${ }^{\circledR} 2000$ (Invitrogen; Thermo Fisher Scientific, Inc.), in order to promote hsa-miR-371-5p expression, according to the manufacturer's protocols. A total of $24 \mathrm{~h}$ post-transfection, the cells were harvested and total RNA and protein were extracted for qPCR and western blot analyses. The sequences were as follows: Hsa-miR-371-5p mimics, 5'-ACU CAAAAGAUGGCGGCACUU-3'; and control mimics, 5'-AGUACUUUUGUGUAGUACA-3'.

Analysis of mesangial cell proliferation. Mesangial cell proliferation was detected using an MTT assay (Sigma-Aldrich; Merck Millipore), according to the manufacturer's protocol. Briefly, 4,000 mesangial cells were cultured in 96-well plates and treated with 10 pmol hsa-miR-371-5p mimics or control mimics. At $0,24,48$ or $72 \mathrm{~h}$ post-transfection, the cells were treated with $30 \mu \mathrm{MTT}(5 \mu \mathrm{g} / \mathrm{ml})$ for $4 \mathrm{~h}$ at $37^{\circ} \mathrm{C}$, then with $150 \mu \mathrm{l}$ dimethyl sulfoxide for $15 \mathrm{~min}$ at $37^{\circ} \mathrm{C}$. The results were determined using a microplate reader (Bio-Rad Laboratories, Inc., Hercules, CA, USA) at a dual wavelength of $450 / 630 \mathrm{~nm}$.

Analysis of mesangial cell apoptosis. Mesangial cells $\left(\sim 8 \times 10^{5}\right)$ were plated into 6-well plates and were transfected with hsa-miR-371-5p mimics or control mimics within $24 \mathrm{~h}$. All mesangial cells, including floating and dead cells, were collected $48 \mathrm{~h}$ post-transfection, were washed twice with 
cold PBS and were treated with $100 \mu 11 \mathrm{X}$ binding buffer (Invitrogen; Thermo Fisher Scientific, Inc.). Subsequently, $5 \mu 1$ Annexin V-fluorescein isothiocyanate (Invitrogen; Thermo Fisher Scientific, Inc.) and $4 \mu 1$ propidium iodide were added to the reactions. The results were analyzed using flow cytometry (Beckman Coulter, Inc., Brea, CA, USA) at $488 \mathrm{~nm}$ excitation within $30 \mathrm{~min}$ of staining, according to the manufacturer's protocols.

Plasmid construction and luciferase activity analysis. PicTar (http://pictar.mdc-berlin.de/) and TargetScan (http://www.targetscan.org/) databases were used to predict the complementary region of hsa-miR-371-5p to HIF-1 $\alpha$. Luciferase reporters were constructed using molecular cloning technology. The target sequence was inserted into the psiCHECK-2 luciferase reporter plasmid (Promega Corporation, Madison, WI, USA) to obtain the psiCHECK-2-HIF-1 $\alpha$ wild type (WT) recombinant reporter vector, which contained the hsa-miR-371-5p binding sequence. The psiCHECK-2-HIF-1 $\alpha$ mutant (MUT) recombinant reporter vector was chemically synthesized by Shanghai GenePharma Co., Ltd.

Luciferase assays were then used to assess whether HIF-1 $\alpha$ was a direct target of hsa-miR-371-5p. Briefly, mesangial cells were cultured in 24-well plates until they reached 70-80\% confluence, when they were transiently co-transfected with $0.2 \mu \mathrm{g}$ WT or MUT reporter plasmid, $10 \mathrm{nmol}$ hsa-miR-371-5p mimics or control mimics using Lipofectamine ${ }^{\circledR} 2000$ (Invitrogen; Thermo Fisher Scientific, Inc.). A total of $48 \mathrm{~h}$ post-transfection, all samples were harvested and analyzed using the Dual-Luciferase Reporter Assay system (Promega Corporation), according to the manufacturer's protocol. Renilla luciferase activity was normalized to Firefly luciferase activity for each experiment. All transfection assays were carried out in triplicate.

Statistical analysis. Statistical analysis was performed using SPSS 16.0 statistical software package (SPSS, Inc., Chicago, IL, USA). The expression levels of hsa-miR-371-5p in LN renal tissues and normal kidney tissues, luciferase assay, cell expression and flow cytometry results were analyzed using Student's t-test. The differences between hsa-miR-371-5p mimics- or control mimics-transfected mesangial cells in the MTT assay were analyzed using one-way analysis of variance with Dunnett's multiple comparison test ( $>2$ groups) or t test (2 groups). $\mathrm{P}<0.05$ was considered to indicate a statistically significant difference.

\section{Results}

Hsa-miR-371-5p expression is downregulated in LN renal tissues. The expression levels of hsa-miR-371-5p were detected in $35 \mathrm{LN}$ renal tissues and 35 non-cancerous normal kidney tissues using RT-qPCR. As shown in Fig. 1, the expression levels of hsa-miR-371-5p were significantly decreased in the LN renal tissues compared with in the non-cancerous normal kidney tissues.

Restoration of hsa-miR-371-5p suppresses human mesangial cell proliferation. Since hsa-miR-371-5p expression was markedly decreased in $\mathrm{LN}$ renal tissues, the present study

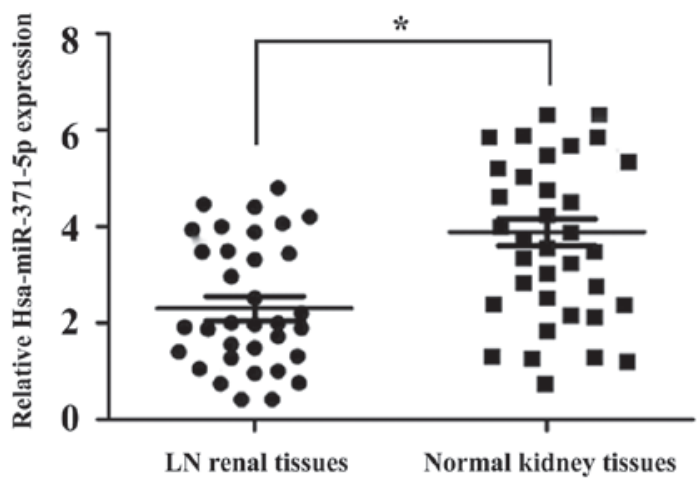

Figure 1. Quantitative polymerase chain reaction analysis of hsa-miR-371-5p expression in LN renal tissues and non-cancerous normal kidney tissues. Data are presented as the mean \pm standard deviation $(n=3)$. ${ }^{*} \mathrm{P}<0.01$ vs. normal kidney tissues. LN, lupus nephritis; miR, microRNA.

hypothesized that low levels of hsa-miR-371-5p may be associated with human mesangial cell growth. To test this hypothesis, hsa-miR-371-5p mimics and control mimics were transfected into human mesangial cells. The relative expression levels of hsa-miR-371-5p were then detected by RT-qPCR. The expression levels of hsa-miR-371-5p were 57.26-fold higher in human mesangial cells transfected with hsa-miR-371-5p mimics, as compared with in cells transfected with control mimics (Fig. 2A).

Mesangial cell proliferation was detected using an MTT assay. The effects of hsa-miR-371-5p overexpression were determined on human mesangial cell proliferation. The results revealed that transfection with hsa-miR-371-5p mimics significantly inhibited human mesangial cell proliferation compared with in the control group (Fig. 2B).

Restoration of hsa-miR-371-5p induces human mesangial cell apoptosis. A flow cytometric assay was performed to examine the apoptotic effects of hsa-miR-371-5p on human mesangial cells. As shown in Fig. 3, $48 \mathrm{~h}$ post-transfection the apoptotic rate of the hsa-miR-371-5p mimics group was $15.06 \%$, as compared with $7.32 \%$ in the control mimics group. These results suggest that overexpression of hsa-miR-371-5p may promote human mesangial cell apoptosis.

$H I F-1 \alpha$ is a direct target of hsa-miR-371-5p. Bioinformatics analyses demonstrated that the HIF-1 $\alpha$ mRNA 3'-UTR contained a potential complimentary binding site to hsa-miR-371-5p (Fig. 4A). In order to verify this prediction, a luciferase assay was conducted in human mesangial cells. Relative luciferase activity was markedly decreased in the hsa-miR-371-5p-transfected human mesangial cells, whereas there was no significant difference in luciferase activity between the hsa-miR-371-5p mimics and control mimics group following co-transfection with the MUT recombinant plasmid (Fig. 4B). These results indicate that HIF-1 $\alpha$ is a direct target gene of hsa-miR-371-5p in human mesangial cells.

HIF-1 $\alpha$ expression is downregulated by hsa-miR-371-5p in human mesangial cells. The effects of hsa-miR-371-5p on the endogenous expression of HIF-1 $\alpha$ were subsequently confirmed in human mesangial cells. The present study aimed 
A

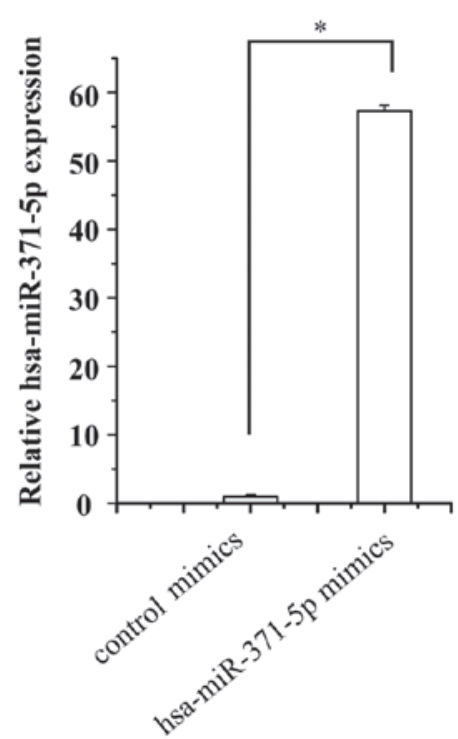

B

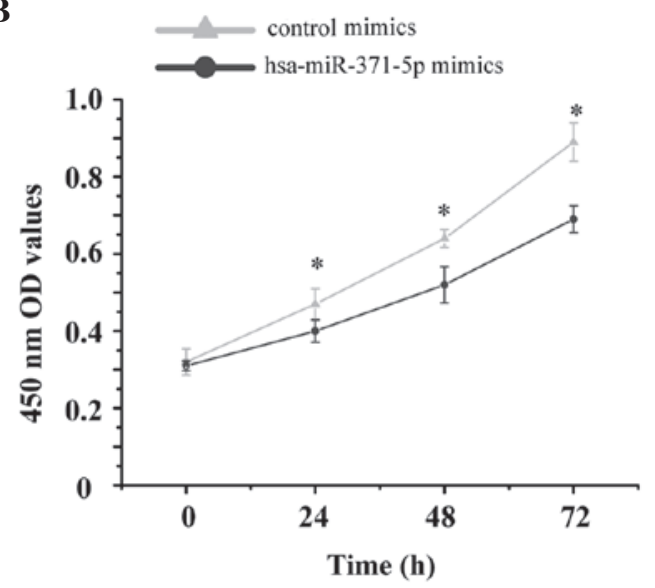

Figure 2. Restoration of hsa-miR-371-5p suppressed human mesangial cell proliferation. (A) Relative expression levels of hsa-miR-371-5p in human mesangial cell post-transfection with hsa-miR-371-5p mimics or control mimics. " $\mathrm{P}<0.001$ vs. control mimics. (B) Cell proliferation of human mesangial cells transfected with hsa-miR-371-5p mimics or control mimics, as determined using the MTT assay. Data are presented as the mean \pm standard deviation ( $\mathrm{n}=3$ ). ${ }^{*} \mathrm{P}<0.05$ vs. hsa-miR-371-5p mimics. miR, microRNA; OD, optical density.

A

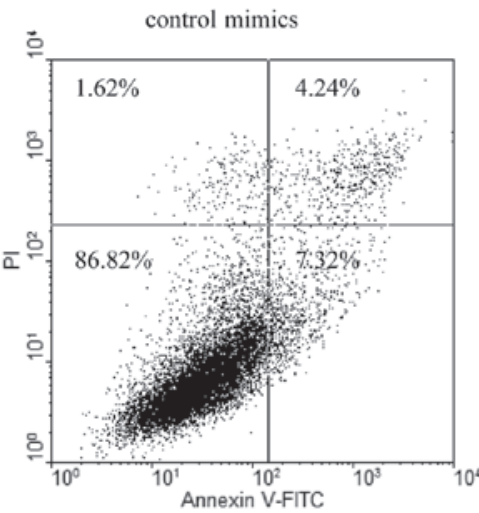

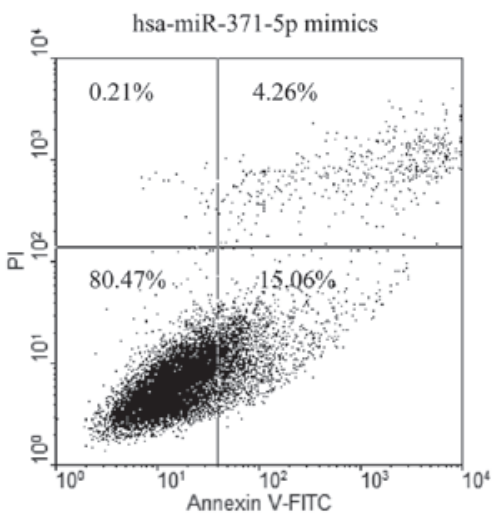

*

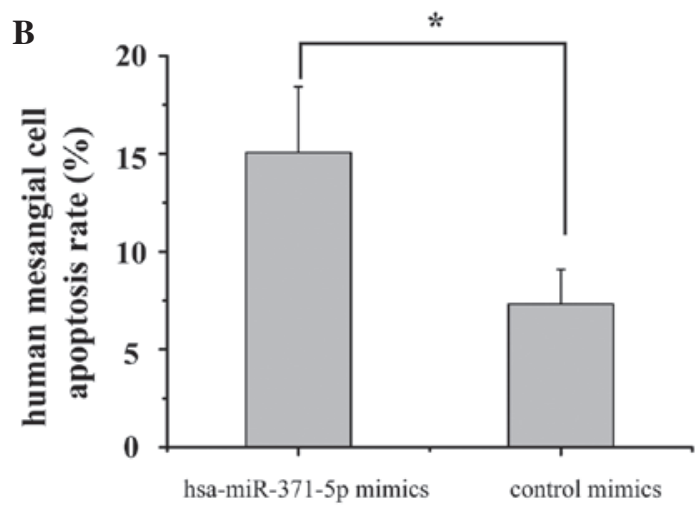

Figure 3. Restoration of hsa-miR-371-5p induced human mesangial cell apoptosis. (A) Human mesangial cell apoptosis was detected by flow cytometric analysis post-transfection with hsa-miR-371-5p mimics and control mimics. (B) The cell apoptotic rate of each group was determined. "P<0.01. vs. control mimics. Data are presented as the mean \pm standard deviation $(n=3)$. miR, microRNA; FITC, fluorescein isothiocyanate; PI, propidium iodide.

to verify whether the mRNA and protein expression levels of HIF-1 $\alpha$ were regulated by hsa-miR-371-5p. Hsa-miR-371-5p mimics were transfected into human mesangial cells using Lipofectamine ${ }^{\circledR} 2000$. RT-qPCR and western blotting results indicated that HIF-1 $\alpha$ expression levels were markedly downregulated in human mesangial cells post-transfection with hsa-miR-371-5p mimics (Fig. 5). These results further confirm that the HIF-1 $\alpha$ is a target gene of hsa-miR-371-5p, 
B

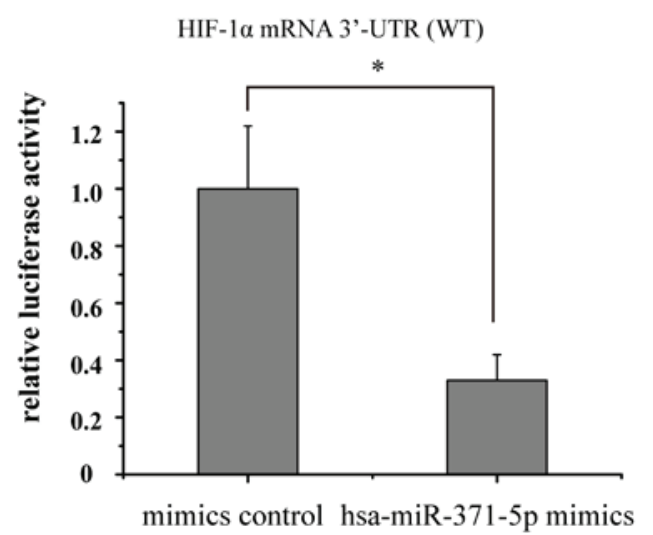

HIF-1 $\alpha$ mRNA 3'-UTR (MUT)

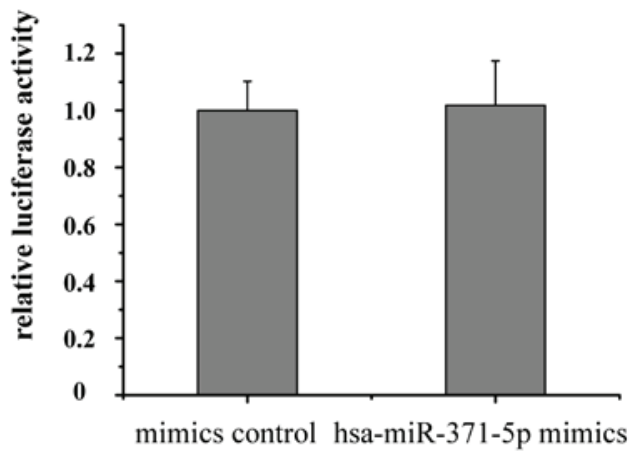

Figure 4. HIF-1 $\alpha$ is a direct target gene of hsa-miR-371-5p. (A) Putative duplex formation between hsa-miR-371-5p and HIF-1 $\alpha$ mRNA 3'-UTR. The seed-recognition sites are underlined in bold-type. (B) Luciferase reporter plasmids contained the HIF-1 $\alpha$ mRNA 3'-UTR binding site, and together with hsa-miR-371-5p mimics or control mimics were co-transfected into human mesangial cells. A total of $48 \mathrm{~h}$ post-transfection, luciferase activity was detected and results were calculated as the ratio of Renilla/Firefly luciferase activities. ${ }^{*} \mathrm{P}<0.005$. vs. control mimics. Data are presented as the mean \pm standard deviation $(\mathrm{n}=3$ ). miR, micoRNA; HIF-1 $\alpha$, hypoxia-inducible factor 1 $\alpha$; 3'-UTR, 3' untranslated region; WT, wild type; MUT, mutant.

and hsa-miR-371-5p can directly modulate HIF-1 $\alpha$ expression in human mesangial cells.

\section{Discussion}

miRNAs act as key regulators of gene expression. It has previously been reported that a single miRNA or several miRNAs serve key roles in regulating cell development and immunoregulation (16). Previous studies have demonstrated that dysregulated miRNA expression in specific cells and tissues may contribute to LN immunopathogenesis (17-19). At present, the possibility of altering miRNA expression for the treatment of $\mathrm{LN}$ remains promising. Studies that alter pathogenic miRNAs have hinted that miRNA-based treatment may be considered a potential therapeutic tool for LN treatment $(20,21)$.

The present study demonstrated that hsa-miR-371-5p expression was downregulated in $\mathrm{LN}$ renal tissues compared with in non-cancerous normal kidney tissues. Several miRNAs have been demonstrated to inhibit cell growth and induce cell apoptosis (22-24). The results of the present study suggested that hsa-miR-371-5p suppressed cell proliferation and promoted the apoptosis of human mesangial cells. These results indicated that hsa-miR-371-5p may be pivotal in the development and progression of LN.

HIF-1 $\alpha$ has been reported to be a vital regulator for the progression of kidney diseases (25). In acute renal injury, HIF-1 $\alpha$ has an important role in adaptation to acute hypoxia via the upregulation of cell-protective HIF-1 $\alpha$ target factors and
A
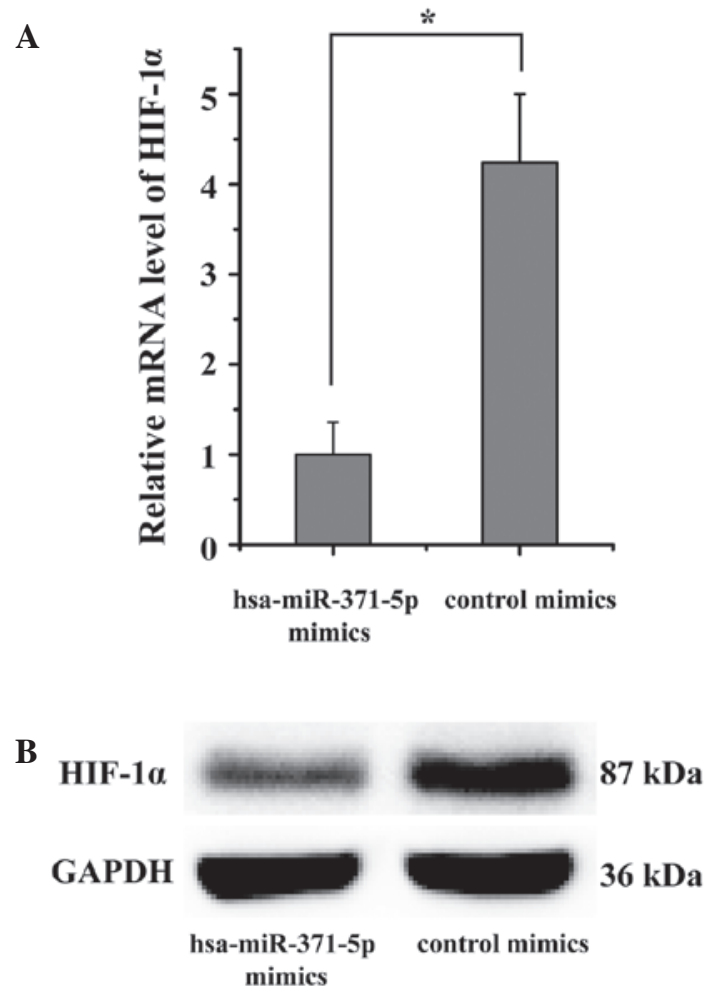

Figure 5. HIF-1 $\alpha$ expression was downregulated by hsa-miR-371-5p in human mesangial cells. (A) Relative mRNA expression levels of HIF-1 $\alpha$ in human mesangial cells post-transfection with hsa-miR-371-5p mimics or control mimics for $48 \mathrm{~h}$. Data are presented as the mean \pm standard deviation $(\mathrm{n}=3) .{ }^{*} \mathrm{P}<0.05$. vs. control mimics. (B) HIF-1 $\alpha$ protein expression was detected by western blot analysis. miR, micoRNA; HIF-1 $\alpha$, hypoxia-inducible factor $1 \alpha$. 
promotion of anaerobic adenosine triphosphate generation (26). In chronic renal injury, chronic hypoxia leads to stabilized HIF-1 $\alpha$ expression and enhances interstitial fibrosis and tissue damage (27,28). Furthermore, Deng et al demonstrated that HIF-1 $\alpha$ was able to promote mesangial cell growth in LN (29). The results of the present study suggested that HIF-1 $\alpha$ is a crucial downstream target of hsa-miR-371-5p in LN. Hsa-miR-371-5p was able to directly bind to the 3'-UTR of HIF-1 $\alpha$, as confirmed by luciferase assay. In addition, restoration of hsa-miR-371-5p was able to markedly downregulate HIF-1 $\alpha$ expression in human mesangial cells. Taken together, these data strongly indicated that hsa-miR-371-5p may alleviate the progression of LN by directly targeting HIF-1 $\alpha$.

In conclusion, the present study suggested that hsa-miR-371-5p was downregulated in LN tissues. Exogenous overexpression of hsa-miR-371-5p inhibited human mesangial cell proliferation and promoted apoptosis through directly targeting HIF-1 $\alpha$. These results indicated that hsa-miR-371-5p may be a key molecule in the development and progression of LN; therefore, hsa-miR-371-5p may be considered a novel and valuable target in the treatment of LN.

\section{Acknowledgements}

The present study was supported by the Youth Science Foundation of Heilongjiang Province of China (grant no. QC2010094).

\section{References}

1. Pan Y and Sawalha AH: Epigenetic regulation and the pathogenesis of systemic lupus erythematosus. Transl Res 153: 4-10, 2009 .

2. Yung S and Chan TM: Autoantibodies and resident renal cells in the pathogenesis of lupus nephritis: Getting to know the unknown. Clin Dev Immunol 2012: 139365, 2012.

3. Lewis EJ and Schwartz MM: Pathology of lupus nephritis. Lupus 14: 31-38, 2005.

4. Aran AA and Putterman C: Treatment of lupus nephritis: Facing the era of immunotherapy. Panminerva Med 50: 235-245, 2008.

5. He L and Hannon GJ: MicroRNAs: Small RNAs with a big role in gene regulation. Nat Rev Genet 5: 522-531, 2004.

6. Bartel DP: MicroRNAs: Genomics, biogenesis, mechanism and function. Cell 116: 281-297, 2004.

7. Davis-Dusenbery BN and Hata A: Mechanisms of control of microRNA biogenesis. J Biochem 148: 381-392, 2010.

8. Kim VN, Han J and Siomi MC: Biogenesis of small RNAs in animals. Nat Rev Mol Cell Biol 10: 126-139, 2009.

9. Mendell JT: MicroRNAs: Critical regulators of development, cellular physiology and malignancy. Cell Cycle 4: 1179-1184, 2005.

10. Calin GA and Croce CM: MicroRNA signatures in human cancers. Nat Rev Cancer 6: 857-866, 2006.

11. Dai Y, Sui W, Lan H, Yan Q, Huang H and Huang Y: Comprehensive analysis of microRNA expression patterns in renal biopsies of lupus nephritis patients. Rheumatol Int 29: 749-754, 2009.
12. Denby L, Ramdas V, McBride MW, Wang J, Robinson H, McClure J, Crawford W, Lu R, Hillyard DZ, Khanin R, et al: miR-21 and miR-214 are consistently modulated during renal injury in rodent models. Am J Pathol 179: 661-672, 2011.

13. Te JL, Dozmorov IM, Guthridge JM, Nguyen KL, Cavett JW, Kelly JA, Bruner GR, Harley JB and Ojwang JO: Identification of unique microRNA signature associated with lupus nephritis. PLoS One 5: e10344, 2010.

14. Wang Y, Yu F, Song D, Wang SX and Zhao MH: Podocyte involvement in lupus nephritis based on the 2003 ISN/RPS system: A large cohort study from a single centre. Rheumatology (Oxford) 53: 1235-1244, 2014.

15. Arocho A, Chen B, Ladanyi M and Pan Q: Validation of the 2-DeltaDeltaCt calculation as an alternate method of data analysis for quantitative PCR of BCR-ABL P210 transcripts. Diagn Mol Pathol 15: 56-61, 2006.

16. Chafin CB and Reilly CM: MicroRNAs implicated in the immunopathogenesis of lupus nephritis. Clin Dev Immunol 2013: 430239, 2013.

17. Zhou H, Hasni SA, Perez P, Tandon M, Jang SI, Zheng C, Kopp JB, Austin H III, Balow JE, Alevizos I and Illei GG: miR-150 promotes renal fibrosis in lupus nephritis by downregulating SOCS1. J Am Soc Nephrol 24: 1073-1087, 2013.

18. Akkina $\mathrm{S}$ and Becker BN: MicroRNAs in kidney function and disease. Transl Res 157: 236-240, 2011.

19. Chen YQ, Wang XX, Yao XM, Zhang DL, Yang XF, Tian SF and Wang NS: Abated microRNA-195 expression protected mesangial cells from apoptosis in early diabetic renal injury in mice. J Nephrol 25: 566-576, 2012.

20. Trionfini P, Benigni A and Remuzzi G: MicroRNAs in kidney physiology and disease. Nat Rev Nephrol 11: 23-33, 2015;

21. Costa-Reis P, Russo PA, Zhang Z, Colonna L, Maurer K, Gallucci S, Schulz SW, Kiani AN, Petri M and Sullivan KE: The role of microRNAs and human epidermal growth factor receptor 2 in proliferative lupus nephritis. Arthritis Rheumatol 67: 2415-2426, 2015.

22. Liu W, Liu H, Li T and Xu G: MicroRNA-490-43p regulates cell proliferation and apoptosis by targeting HMGA2 in osteosarcoma. FEBS Lett 589: 3148-3153, 2015.

23. Luo H, Guo W, Wang F, You Y, Wang J, Chen X, Wang J, Wang Y, Du Y, Chen X, et al: miR-1291 targets mucin 1 inhibiting cell proliferation and invasion to promote cell apoptosis in esophageal squamous cell carcinoma. Oncol Rep 34: 2665-2673, 2015.

24. Zhang S, Zhang C, Liu W, Zheng W, Zhang Y, Wang S, Huang D, Liu X and Bai Z: MicroRNA-24 upregulation inhibits proliferation, metastasis and induces apoptosis in bladder cancer cells by targeting CARMA3. Int J Oncol 47: 1351-1360, 2015.

25. Mao $S$ and Huang $S$ : The signaling pathway of hypoxia inducible factor and its role in renal diseases. J Recept Signal Transduct Res 33: 344-348, 2013.

26. Fähling M, Mathia S, Paliege A, Koesters R, Mrowka R, Peters H, Persson PB, Neumayer HH, Bachmann $S$ and Rosenberger C: Tubular von hippel-lindau knockout protects against rhabdomyolysis-induced AKI. J Am Soc Nephrol 24: 1806-1819, 2013

27. Haase VH: Hypoxia-inducible factor signaling in the development of kidney fibrosis. Fibrogenesis Tissue Repair 5 (Suppl 1): S16, 2012.

28. Kimura K, Iwano M, Higgins DF, Yamaguchi Y, Nakatani K, Harada K, Kubo A, Akai Y, Rankin EB, Neilson EG, et al: Stable expression of HIF-1alpha in tubular epithelial cells promotes interstitial fibrosis. Am J Physiol Renal Physiol 295: F1023-F1029, 2008.

29. Deng W, Ren Y, Feng X, Yao G, Chen W, Sun Y, Wang H, Gao X and Sun L: Hypoxia inducible factor-1 alpha promotes mesangial cell proliferation in lupus nephritis. Am J Nephrol 40: 507-515, 2014. 